\title{
REPORT ON THE FIRST VLHC PHOTON STOP CRYOGENIC DESIGN EXPERIMENT
}

\author{
M. Geynisman, P. Bauer, R. Bossert, C. Darve, K. Ewald, A. Klebaner, \\ P. Limon, A. Martinez \\ Fermi National Accelerator Laboratory* \\ Batavia, Illinois, 60510, USA
}

\begin{abstract}
As part of Fermilab’s study of a Very Large Hadron Collider (VLHC), a water-cooled photon stop was proposed as a device to intercept the synchrotron radiation emitted by the high-energy proton beams in the high-field superconducting magnets with minimal plugcooling power. Photon stops are radiation absorbers operating at room temperature that protrude into the beam tube at the end of each bending magnet to scrape the synchrotron light emitted by the beam one magnet up-stream. Among the technological challenges regarding photon stops is their cryo-design. The photon stop is water-cooled and operates in a cryogenic environment. A careful cryo-design is therefore essential to enable operation at minimum heat transfer between the room temperature sections and the cryogenic parts. A photon stop cryo-design was developed and a prototype was built. This paper presents the results of the cryogenic experiments conducted on the first VLHC photon-stop prototype.
\end{abstract}

\section{INTRODUCTION}

The next generation of large hadron colliders, following CERN's Large Hadron Collider, such as the proposed VLHC [1] in its high-field magnet stage (VLHC2), will be limited in energy and luminosity by the considerable synchrotron-radiation (SR) power emitted by the beams steered through the high-field magnets. The SR power radiated by the VLHC2, as proposed in [1], is $5 \mathrm{~W} / \mathrm{m}$ per beam. This represents a $\sim 50$ fold increase of SR power per unit length from the LHC level, and together with the 10 times larger circumference of the VLHC, would make it very costly to absorb the SR at the low temperature of the cryo magnets. It is therefore necessary to develop solutions for a cost effective removal of the SR power. One such solution $[1,2]$ is to insert a beam-screen into

\footnotetext{
* Work supported by the U.S. Department of Energy under contract No. DE-AC0276CH03000
} 
the magnet bore operating at a higher temperature than the magnet cold mass, such as in the LHC. Given the SR load mentioned above, the optimum beam-screen temperature, i.e. the temperature at which the cooling cost at the plug is minimized, is $\sim 100 \mathrm{~K}$ to limit radiation heat transfer between the beam screen and the cryo magnet. The beam screen cold bore assembly also serves as a cryopump to remove the gases photo-desorbed from the photon stop tip by the SR.

A better solution is to use water-cooled photon stops that protrude into the beam tube at the end of each bending magnet and scrape off the synchrotron light at room temperature $[3,4]$. A careful cryo-design for such photon stop (PS) is essential to enable operation at minimum heat transfer between the room-temperature radiation absorber and the parts at cryogenic temperatures. PS cryo-design was developed [5] and a first PS prototype was built. The PS prototype consists of a water-cooled copper tube with a copper absorber at its tip, surrounded by an inner and an outer PS thermal shield. The shielded absorber is assembled into a mock-up of the magnet inter-connect consisting of a short $(\sim 1 \mathrm{~m})$ section of dummy cold mass and beam screen. The 100-K beam screen (which, according to the current PS design, would be limited to the magnet-interconnect region only) serves as a thermal shield for the cold mass, absorbing the radiation emitted (or reflected) from the room-temperature photon-stop tip.

\section{DESIGN}

As shown in FIGURE 1 the absorber (or PS) enters perpendicularly into the dummy cold-mass / beam-screen assembly emulating a real accelerator magnet interconnect environment. In FIGURE 1 only the outer PS shield bellows are visible and the lower part of the inner PS shield is not. The inner PS thermal shield is actively cooled to $\sim 80 \mathrm{~K}$ throughout the bottom half and negotiates a temperature drop from room temperature to $\sim 80 \mathrm{~K}$ through bellows forming its upper half. The lower, $80 \mathrm{~K}$ part of the inner PS shield in turn is surrounded by the outer PS shield bellows, which negotiate a temperature drop from $\sim 80 \mathrm{~K}$ at the top to $\sim 5 \mathrm{~K}$ at the bottom.
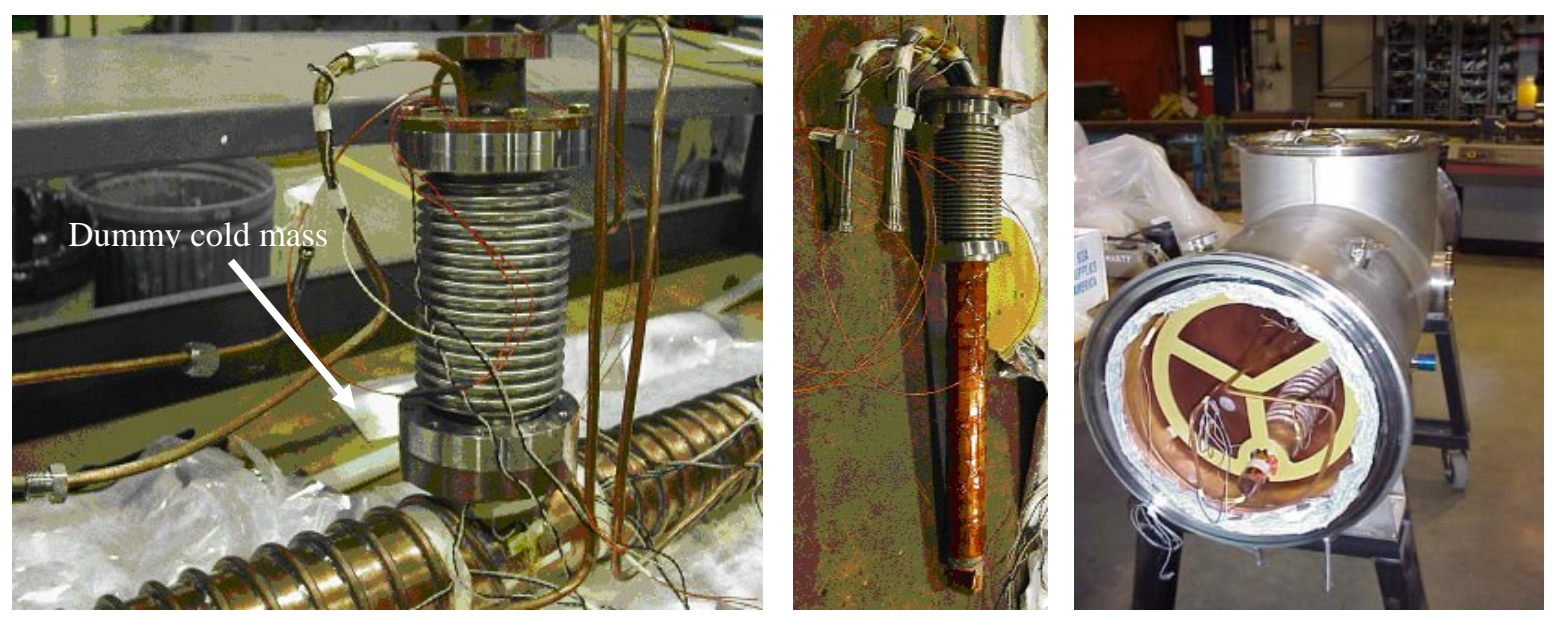

FIGURE 1. Photon-Stop prototype for the first cryogenic design experiment. Left: dummy cold-mass mockup with PS shield assembly (vertical). The inner PS shield is within the outer PS shield bellows. The tubes supplying the inner PS shield with $~ 80 \mathrm{~K}$ gaseous He can be seen. Middle: PS absorber with inner PS shield top bellows and water pipes. The PS absorber is instrumented with safety heaters. Right: Cryostat housing the PS prototype. The photo shows one of the G10 spiders supporting the PS prototype in the cryostat thermal shield. 


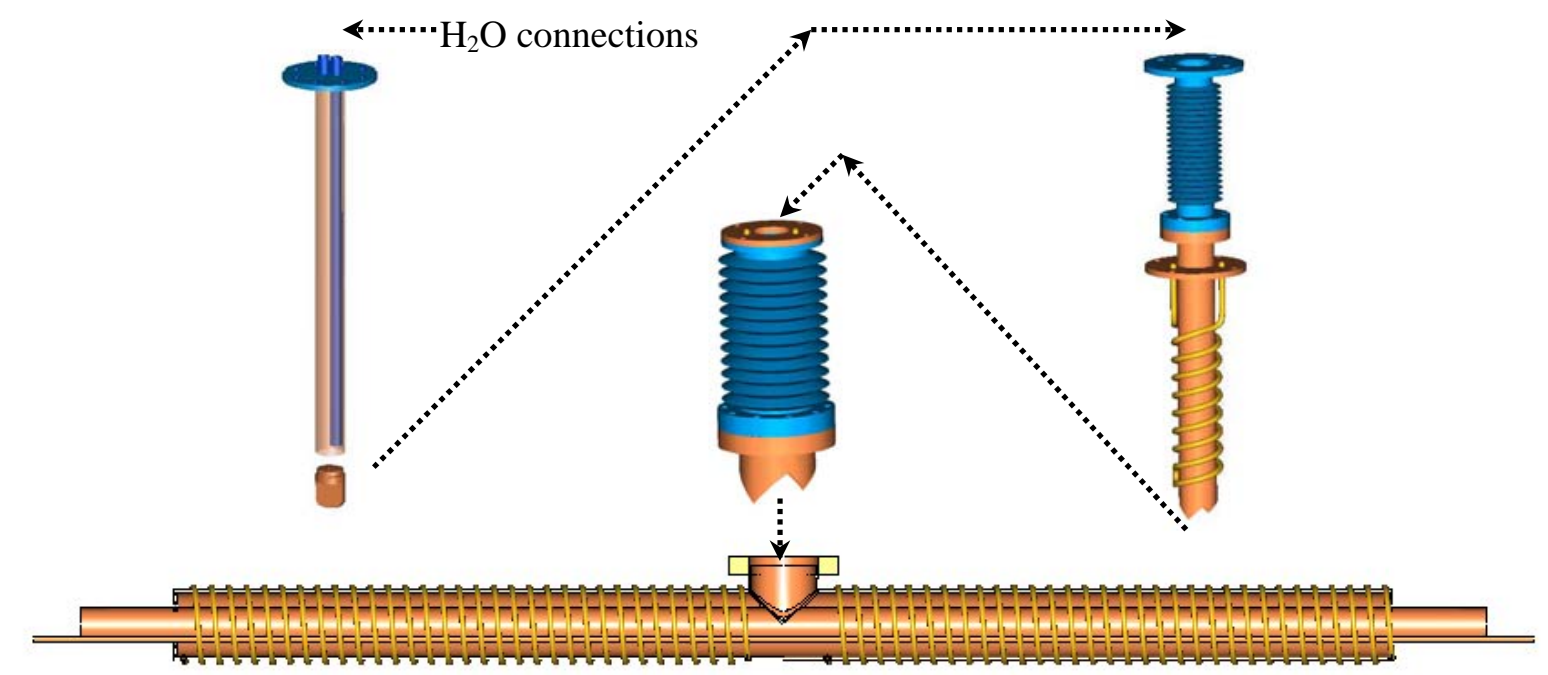

FIGURE 2. PS absorber (left), outer PS shield (middle), inner PS shield (right), PS beam screen (bottom).

FIGURE 2 shows the main components of the absorber assembly. The absorber is a 0.0375-m Ø, 0.5-m long copper tube with a nose piece (the real absorber) welded to it at one end and a flange welded to it on the other end. At the seam between the nose piece and the copper tube are three G10 pins distributed around the tube perimeter. The absorber is in direct thermal contact with the inner PS shield only through the G10 centering pins.

The inner PS thermal shield consists of a 0.038-m Ø copper tube with a brazed cooling spiral and 0.184-m long stainless-steel bellows connected by a mixed steel/copper flange. At the bottom the inner shield is welded to the beam screen. At the top end the shield is at room temperature and bolted to the top flange of the water-cooled PS. The outer PS shield is composed of a 0.076-m Ø copper saddle and flange, stainless steel flanges and a 0.076-m $\varnothing, 0.184-\mathrm{m}$ long stainless-steel bellows. On top it is bolted to a copper flange, which is welded to the inner shield mid-section. At the bottom the outer PS shield is welded to the dummy cold mass. The dummy cold mass is a 1.52-m long, 0.076-m $\varnothing$ copper tube cooled with a spiral 0.00635-m $\varnothing$ copper tubing containing liquid helium at $\sim 5 \mathrm{~K}$. It also contains a beam screen running along its entire length as a shield from the radiation emitted by the photon-stop tip. The beam screen is cooled to $\sim 80 \mathrm{~K}$ with helium gas flowing in a single pass cooling tube soldered along the screen. The beam screen is supported in the dummy cold mass tube by two small G10 spiders, which are designed to minimize thermal contact and wedged between cold bore and beam screen in the ends. The complete photon-stop cryo-assembly is mounted into a vacuum vessel (see FIGURE 1), which has its own $80 \mathrm{~K}$ radiation shield and throughput connections for water, liquid helium and cold gaseous helium. The PS assembly is installed into the cryostat using large G-10 spiders (0.0064-m thickness, 1-m $\varnothing, 0.0255-\mathrm{m}$ width, shape: Y in circle). The helium transfer lines enter the cryostat through the top access window. The water tubes enter through small side-ports in the middle of the cryostat.

\section{EXPERIMENTAL SETUP}

The experimental setup is shown in FIGURE 3. The test was conducted at the Fermilab Cryogenic Test Facility. The facility contains four $600 \mathrm{~W}$ (at 4.5K) refrigerators. One of the refrigerators was configured to supply gas helium at $40-80 \mathrm{~K}$ for the thermal shields and $5 \mathrm{~K}$ (and higher) for the dummy cold mass. The refrigerator is a CTI design 
with STAR-type heat exchangers, liquid-nitrogen pre-cool and CTI reciprocatingexpansion engines. The refrigerator was coupled to a $4-\mathrm{m}^{3}$ helium dewar for the production output and regeneration of boil-off, and to a $225-\mathrm{m}^{3}$ warm-helium storage buffer. The refrigerator and the experimental setup were operated and monitored with a Siemens Advanced Process Automation and Controls System (APACS). The refrigerator capacity was limited to $\sim 1 \mathrm{~g} / \mathrm{s}$ at $5 \mathrm{~K}$ plus $1 \mathrm{~g} / \mathrm{s}$ at $30 \mathrm{~K}$ during mixed-mode operation. That necessitated running the experiment in a batch mode when helium flows to the experiment were higher than $1 \mathrm{~g} / \mathrm{s}$. A distribution valve box (BR), vacuum-insulated U-tubes and hoses, and a transitional vertical vacuum vessel were used to route the helium flow from the refrigerator to the PS cryostat. Cold 5-K helium was drawn from the exhaust of the "wet" expansion engine (WE). Due to inadequate design of the transitional elements, the actual temperature of cold helium supplied to the dummy cold mass could not be below 12 $\mathrm{K}[6]$. 30-K helium was drawn from the inlet of the "dry" expansion engine (DE) and could be warmed up with an $800 \mathrm{~W}$ heater (HTR1). The setup included a control valve (EVWHS) to control the 30-K helium mass flow to the PS experiment and a control valve (EVCHR) to control cold helium flow to the dummy cold mass. Pressures, temperatures and flows were measured. Four Cernox ${ }^{\mathrm{TM}}$ temperature sensors (CX-1050-SD) were distributed along the dummy cold mass tube, measuring temperatures from $4 \mathrm{~K}$ to $300 \mathrm{~K}$. Six platinum temperature sensors (PT102) were distributed along the 80-K shields path, measuring temperatures from 30 to $300 \mathrm{~K}$. The sensitivity of the CX-1050-SD and PT102 were on the order of $10^{-3} \Omega / \mathrm{K}$ at $5 \mathrm{~K}$ and $0.4 \Omega / \mathrm{K}$ at $70 \mathrm{~K}$, respectively [6].

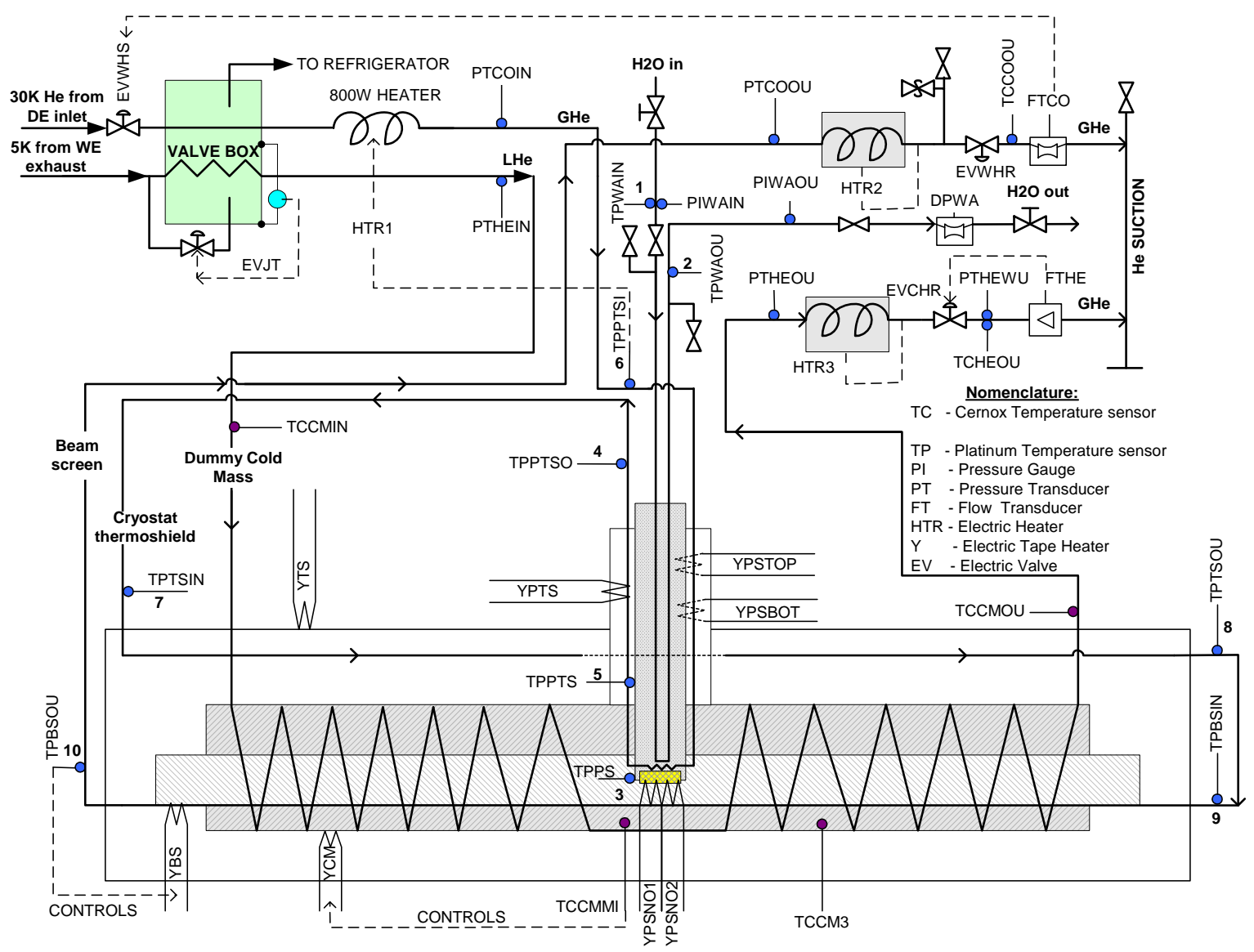

FIGURE 3. Experimental setup of the photon stop test. 
The temperature sensors were read out with the four-wire measurement technique to minimize the influence of the wire resistance. Manganin wires were used to limit heat load from the ambient to the low temperature system. In the case of the Cernox ${ }^{\mathrm{TM}}$ sensors the current was controlled to maintain a constant voltage and limit the self-heating. Lake Shore Cryotronics, Inc. individually calibrated each Cernox ${ }^{\mathrm{TM}}$ sensor and provided $6^{\text {th }}$ order Chebychev polynomials calibration fits covering four temperature ranges. Temperature sensors were mounted to the PS with varnish (LakeShore VGE1731) and epoxy (Stycast $^{\mathrm{TM}}$ ). An error of $0.02 \mathrm{~K}$ was calculated for temperatures measured by the Cernox $^{\mathrm{TM}}$.

The helium flow through the dummy cold mass was measured with a turbine flowmeter. The helium gas for the shields and water flow were measured with Venturi flowmeters. The calculations for the helium mass flows were adjusted for temperature and pressure to compensate for density variations.

Eight tape heaters were affixed with bonding agent at strategic locations to either emulate the synchrotron radiation heat load on the tip of the PS, or as part of the anti-freeze precaution for the PS, as well as for the residual heat load measurements using the so called "heater technique" (described later). Heaters were made of Nickel-Steel alloy foil encapsulated in Kapton ${ }^{\mathrm{TM}}$ foil. The alloy is such that the resistance change at low temperature is $<1 \%$.

The controls and data acquisition system was based on the industrial Siemens-Moore APACS automation system. APACS allowed non-linear scaling of the raw data, automatic regulation of controlled variables, e.g. flow rates and temperatures, and safety interlocks. The safety measures included a set of configurable alarms, automatic closing of all helium supply valves in the case of loss of water flow or the safety heaters, and the possibility to blow-out water from the stop using compressed air. APACS also provided a synchronous transfer of all data at $1 \mathrm{~Hz}$ rate to the remote server for storage. That allowed for unlimited data collection and analysis.

\section{MEASUREMENTS GOALS AND PRINCIPLES}

The goals of the PS cryo-test were a) to prove feasibility of the PS cryo design and operation; b) to measure components' heat loads due to thermal cross-talk, hence to determine the efficiency of the PS technology; c) to validate the theoretical model.

To measure the residual heat load on the dummy cold mass, inner PS shield, beam screen, and cryostat thermal shield, each of those sub-systems was equipped with temperature sensors on the inlet and outlet of its cooling circuit. The sub-system residual heat load was derived from inlet and outlet temperature for each equilibrium configuration using equation (1).

$$
\left(Q_{e l}\right)+Q_{0}=\dot{m} C_{p}\left(T_{\text {out }}-T_{\text {in }}\right)
$$

where: $\dot{m}$ is the mass flow of helium cooling the sub-system of interest, $C_{p}$ the cryogen specific heat, and $\left(T_{\text {out }}-T_{\text {in }}\right)$ the measured temperature difference across the sub-system cooling circuit.

To crosscheck the results, another method, the so-called "heater method" was used. The method employed electric heaters attached to each sub-system and powered with a known amount of electrical power $Q_{e l}$ (see equation (1)), typically 10, 25 and $50 \mathrm{~W}$. The 
residual heat load $Q_{0}$ could then be extrapolated from the heat loads measured at varying electrical heating powers [6].

Several configuration cases were studied in which the following parameters were varied: a) operating temperatures in the shield circuit $(45-77 \mathrm{~K})$; b) operating temperature in the cold mass circuit (15-25 K); c) temperature of the PS water-cooling circuit (80 - 300 $\mathrm{K})$; d) temperature of the PS nose $(80-400 \mathrm{~K})$. The dummy cold mass circuit temperature was controlled by varying the helium mass flow $(0.3-1.2 \mathrm{~g} / \mathrm{s})$. The supply helium temperature was limited by transfer losses such that temperature in the dummy cold mass circuit could not be lower than $12 \mathrm{~K}$. Thermal equilibrium was typically reached after $10-$ 20 min. Equilibrium temperatures and many other sub-system parameters were recorded and the residual heat load $Q_{o}$ was determined. In the case of the dummy cold mass, the residual heat load was measured with two methods such as described above.

\section{RESULTS AND ANALYSIS}

Seven cases were studied in which the operational parameters were varied as described above [6]. Table 1 summarizes the parameters and the residual heat loads measured on the dummy cold mass and the inner PS shield. The following observations were made: a) the PS temperature affects every component of the cryostat, i.e. the residual heat load measurements on the cold-mass as well as the shield circuits indicate an increase of the static heat load with the PS absorber temperature; b) variations in the $80 \mathrm{~K}$ shields circuit temperature between $\sim 45 \mathrm{~K}$ and $\sim 80 \mathrm{~K}$ result in a 5-fold cold mass residual heat load increase; c) reduction in the temperature of the cold mass circuit from $\sim 25 \mathrm{~K}$ to $\sim 15 \mathrm{~K}$ results in the residual heat load increasing $\sim 5$-fold.

A disagreement, as large as $20 \%$, between the residual heat loads on the dummy cold mass derived from the two methods was found. Possible explanations for this discrepancy are: a) that the heater power, $Q_{e l}$, applied on electric tape heater YCM, was chosen too large compared to $Q_{0}$, inducing extrapolation errors in conjunction with the measurement uncertainty and b) that the YCM heater was mounted in close proximity to the cold bore cooling circuit outlet temperature sensor (TCMMOU), thus affecting its readings.

FIGURE 4 shows the comparison between experimental and expected results. The expected modes of heat transfer were based on radiation and conduction from and to each sub-system at measured temperature levels. Material properties, surfaces and contact area, and temperatures were modeled to calculate the expected residual heat loads on the different sub-system. The main heat-transfer contributions (in the order of importance) were: a) radiation and conduction from the ambient to the cryostat thermal shield; b) radiation from the PS absorber to the inner PS shield; c) radiation from the cryostat thermal shield and in particular end caps to the surrounding shields and dummy cold mass; d) conduction from the beam screen to the dummy cold mass through two small G10 spiders; e) conduction from room temperature to the dummy cold mass through the bellows system; f) conduction from the PS nose to the inner PS shield through three G10 pins located in the annular gap; g) conduction from the cryostat thermal shield to the beam screen through two large G10 spiders; $h$ ) radiation from the beam screen to the dummy cold mass.

Results in FIGURE 4 indicate that the residual heat loads measured on the dummy cold mass and inner PS shield were much larger than expected. One of the PS design goals, for example, is that the residual heat load on the cold mass is less than $1 \mathrm{~W}$. This goal was not reached. Additionally, the temperature readings in the $80 \mathrm{~K}$ beam screen circuits and 
cryostat thermal shield were not always consistent with the direction of flow of the coolant such that the residual heat loads could not be derived with confidence.

TABLE 1. Summary of results in all experimental cases of the photon stop test.

\begin{tabular}{lcccccccc}
\hline & PS nose & \multicolumn{3}{c}{ cold mass } & \multicolumn{3}{c}{ 80 K shields } \\
\hline & $\mathrm{T}(\mathrm{K})^{1}$ & $\mathrm{~T}(\mathrm{~K})$ & $\mathrm{m}(\mathrm{g} / \mathrm{s})$ & $\mathrm{Qo}(\mathrm{W})$ & $\mathrm{Qo}(\mathrm{W})^{2}$ & $\mathrm{~T}(\mathrm{~K})^{1}$ & $\mathrm{~m}(\mathrm{~g} / \mathrm{s})$ & Qo PTS $(\mathrm{W})$ \\
\hline CASE B & 271.00 & 14.64 & 0.977 & 10.71 & 9.87 & 77.00 & 1.008 & 16.64 \\
CASE D & 271.00 & 23.59 & 0.296 & 2.54 & 2.77 & 45.00 & 1.034 & 15.28 \\
CASE E & 360.00 & 22.29 & 0.307 & 2.99 & 2.97 & 45.00 & 1.010 & 19.76 \\
CASE H & 271.00 & 24.54 & 0.323 & $1.78 *$ & $\mathrm{~N} / \mathrm{A}$ & 45.00 & 1.007 & 14.92 \\
CASE I & 376.00 & 25.13 & 0.299 & 3.60 & 3.44 & 46.00 & 1.017 & 19.25 \\
CASE J & 83.00 & 24.00 & 0.302 & 1.13 & 0.75 & 43.00 & 0.998 & 9.37 \\
CASE K & 85.00 & 13.08 & 1.006 & 1.15 & 0.55 & 43.00 & 1.062 & 9.06 \\
\hline
\end{tabular}

1 - Average temperature; 2 - Measured with "header method;” * - Result not validated by "heater method.”

The mounting method of platinum RTD PT102 temperature sensors was proven to be inappropriate for this type of measurement. The error bars for the data in FIGURE 4 were derived from observed temperature fluctuations in equilibrium conditions. The observed temperature fluctuations were 4 to 10 times larger than the expected $0.02 \mathrm{~K}$ error of the Cernox $^{\mathrm{TM}}$ temperature measurement. These fluctuations are believed to be induced by process variable variations, such as variations in the flow [6].

In an effort to understand the discrepancies between the experimental and expected residual heat loads, a theoretical mathematical model was used. The theoretical model was built to differentiate sources of heat transfer, like conduction and radiation, which cannot be set apart in measurements. The post-experimental mathematical analysis allowed simulating additional heat loads due to radiation and conduction for the existing subsystems. Using these additional terms we could explain the discrepancies between the original model and experimental results. The mathematical model is detailed in [6]. We estimated that 30 times more radiation to the dummy cold mass would explain the measured results.

Those additional radiation sources affecting the dummy cold mass could have been the water tubes supplying room temperature water to the PS absorber since, inadvertently, they were not covered with MLI, as well as, the end caps that were estimated to be $20 \mathrm{~K}$ warmer than expected due to insufficient cooling of the cryostat thermal shield. In addition, 10 times more conduction to the cold bore than expected would be needed to explain the experimental results. This could be due to excessive conduction through the outer PS shield and/or bad thermalization of its upper warm flange. The analysis of the experimental data also hints toward the occurrence of a direct conductive contact between beam screen and cold mass as well as between the photon-stop insert and the photon-stop thermal shield.
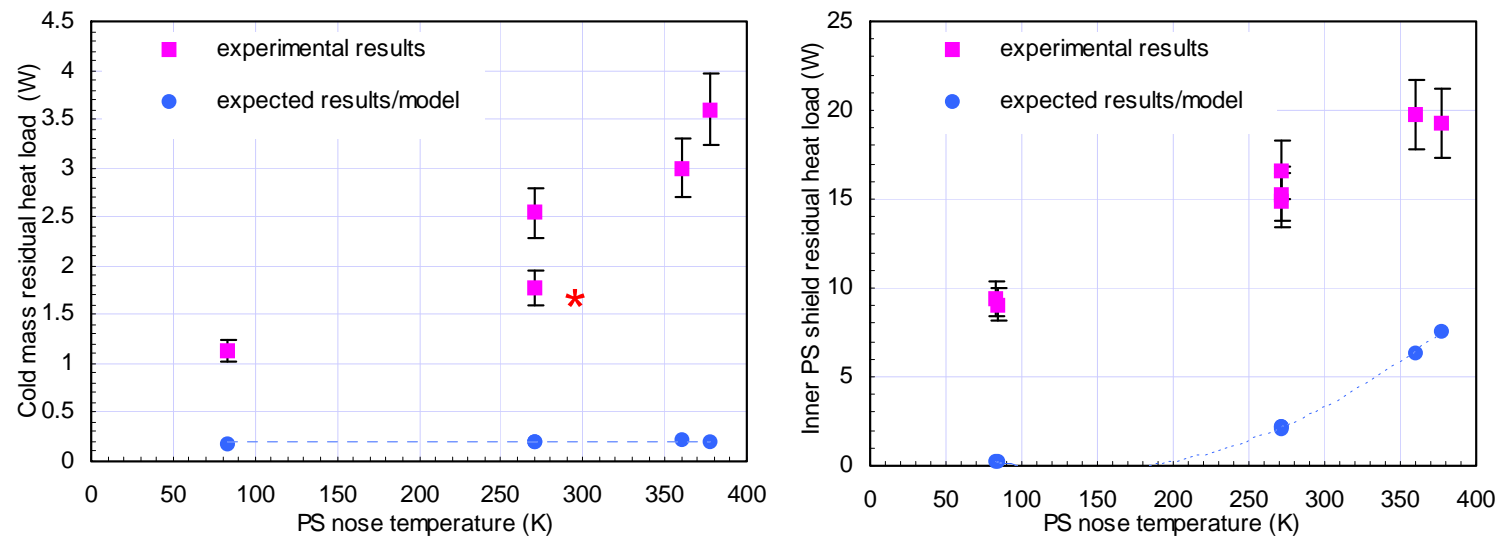
FIGURE 4. Measured and expected residual heat loads for: $\mathrm{Q}_{0}$ of the cold mass at $24 \mathrm{~K}$ (left); $\mathrm{Q}_{0}$ of the inner PS shield as function of temperature of PS nose (right). *- Result not validated by "heater method."

The former could be the result of sagging of the beam screen assembly in the dummy cold mass under the weight of the photon stop. The latter could be the result of a build-up of insulation (associated with safety heaters installation) beyond the allowed thickness given by the $\sim 0.0015$-m gap between the photon stop insert and the inner PS shield.

\section{CONCLUSIONS}

The first photon stop cryogenic test was conducted at Fermilab during December 2002 and January 2003. All circuits were reliably operated for over 4 weeks, and therefore the major goal of this experiment was achieved. Two methods were used to measure residual heat loads, showing $20 \%$ agreement and providing partial results. The goal of residual heat load of less than $1 \mathrm{~W}$ on the dummy cold mass (magnet cold bore simulation) could not be reached. The experimental data was not conclusive enough to determine what exactly caused the additional heat loads in the system. A mathematical model was used to understand the sources of discrepancies. From this model results, it is very likely that these discrepancies were partially the result of spurious heat loads that were accidentally introduced into the system. These spurious or accidental heat loads were: a) radiation from the water tubes leading to the photon stop, which had not been shielded with MLI; b) radiation from ambient due to insufficient cooling of the cryostat thermal shield (particularly in the end-caps); and possibly c) conduction from the beam screen to the dummy cold mass due to the copper pipe sagging. Summarizing, it has to be noted that one of the most important aspects of this experiment was a valuable learning experience that will allow considerable improvement to the design and test procedures for the next generation of photon stop prototypes.

\section{ACKNOWLEDGMENTS}

We thank Carl Staples, Roger Milholland, Greg Johnson, Gerry Szabo and Mark Nylund for technical assistance.

\section{REFERENCES}

1. P. Limon et al., "Design Study for a Staged Very Large Hadron Collider," Fermilab-TM-2149, June 2001

2. C. Darve et al., "VLHC beam-screen cooling”, FNAL, TD-01-005, Feb. 2001.

3. P. Bauer et al., "Synchrotron Radiation Issues in the VLHC," Proceedings to the Particle Accelerator Conference 2001, Chicago, Sept. 2001.

4. P. Bauer et al., "Synchrotron Radiation Absorbers for Hadron Colliders," Proceedings of the European Particle Accelerator Conference, Paris, France, June 2002.

5. P. Bauer et al., "A Photon Stop for the VLHC-2, Engineering Design 1," FNAL, TD-01-023, April 2001.

6. P. Bauer et al., "Report on the First Cryogenic Photon Stop Experiment,” FNAL TD-03-021, May 2003. 